\title{
Oncogenic miR-744 promotes prostate cancer growth through direct targeting of LKB1
}

\author{
MINGLEI ZHANG ${ }^{1}$, HAI LI ${ }^{2}$, YUN ZHANG ${ }^{2}$ and HONGYAN LI ${ }^{2}$ \\ Departments of ${ }^{1}$ Orthopedics and ${ }^{2}$ Urology, China and Japan Union Hospital of Jilin University, \\ Changchun, Jilin 130000, P.R. China
}

Received December 26, 2017; Accepted October 25, 2018

DOI: $10.3892 /$ ol.2018.9822

\begin{abstract}
Prostate cancer (PCa) is one of the most common malignancies worldwide, and with a limited number of treatments for this type of cancer, its incidence is rapidly increasing. Patients presenting with $\mathrm{PCa}$ are likely to experience disease recurrence, which represents a considerable clinical challenge. MicroRNAs (miRNAs) have been widely characterized as a critical regulator in a number of types of cancer, including PCa. miRNA-744 (miR-744) has been reported to be involved in cancer regulation; however, its role in PCa remained poorly understood. In a recent study, it was demonstrated that miR-744 was overexpressed in prostate tissue from PCa patients when compared with the surrounding tissues, and knockdown of miR-744 resulted in reduced cell growth. In addition, an increased population of apoptotic cells was detected upon miR-744 knockdown, together with a decrease in cell proliferation. Cell cycle analysis demonstrated a higher number of cells in the G1 phase and lower numbers in the $\mathrm{S}$ phase following miR-744 silencing. The levels of key proteins involved in cell cycle progression (cyclin D1, cyclin-dependent kinase 4, and proliferating cell nuclear antigen) were increased, whereas those proteins responsible for cell cycle inhibition (cyclin-dependent kinase inhibitor p21) were decreased. The tumor suppressor liver kinase B1 (LKB1) was revealed to be a potential target of miR-744, suggesting its potential mechanism of action. LKB1 levels were negatively correlated with miR-744, and LKB1 was indicated to be a direct target of miR-744. Furthermore, it was revealed that by targeting LKB1, miR-744 may regulate adenosine monophosphate-activated protein kinase (AMPK); the AMPK signaling pathway was activated by miR-744 knockdown, with subsequent inhibition of the mammalian target of rapamycin
\end{abstract}

Correspondence to: Professor Hongyan Li, Department of Urology, China and Japan Union Hospital of Jilin University, 126 Xiantai Street, Changchun, Jilin 130000, P.R. China

E-mail: lihongyan006@163.com

Key words: prostate cancer, microRNA-744, liver kinase B1, adenosine monophosphate-activated protein kinase signaling pathway, cell growth
(mTOR) signaling pathway. Taken together, these results demonstrated that miR-744 promoted cell growth through the AMPK signaling pathway, by targeting LKB1. The present study revealed a novel insight into the biological function of miR-744 in PCa, and that miR-744 may be a potential therapeutic target.

\section{Introduction}

Prostate cancer (PCa) is one of the most common, life-threatening malignancies among men worldwide (1). The incidence of PCa continues to rise, particularly in developing countries $(2,3)$. Although a number of therapeutic strategies have been developed to treat PCa (including surgery), there remains a high incidence of relapse following primary therapy (4). The involvement of numerous genes in the regulation of $\mathrm{PCa}$ is increasingly apparent (5-7). However, the pathogenesis and progression of this malignancy remains to be fully elucidated, and new therapeutic targets require investigation.

MicroRNAs (miRNAs) are a class of 20-25 nt non-coding RNA transcripts that negatively regulate gene expression by directly binding to the 3'-untranslated region (UTR) of target mRNAs (8). The altered expression of miRNAs, and their contribution to the carcinogenesis and progression of various human cancer types, including PCa, is well characterized (9-11). miR-128 directly targets stem cell regulatory factors, including polycomb complex protein BMI-1 (BMI-1), homeobox protein NANOG, and TGF- $\beta$ receptor type-1 to suppress PCa (12). Downregulation of miR-221, miR-30d and miR-15a contributes to the pathogenesis of PCa by targeting BMI-1 (13). Serum analysis demonstrated that miR-103, miR-125b and miR-222 may serve as biomarkers to monitor therapeutic efficacy in $\mathrm{PCa}$ (14).

In the present study, miR-744, which has been implicated in various human cancer types (15-18), was investigated; miR-744 increases the tumorigenicity of pancreatic cancer by activating the Wnt/ $\beta$-catenin pathway, and elevated serum miR-744 predicts poor prognosis in patients with nasopharyngeal carcinoma. miR-744 functions as a proto-oncogene in nasopharyngeal carcinoma through transcriptional regulation of Rho GTPase-activating protein 5 (ARHGAP5). However, little is known of the biological function of miR-744 in PCa.

In the present study, the biological function and molecular mechanism of miR-744 in PCa were investigated. It was identified that miR-744 was upregulated in PCa tissue when compared to 
the adjacent tissues. Silencing of miR-744 resulted in the inhibition of cell growth, and an increased population of apoptotic cells was identified following miR-744 knockdown. The knockdown of miR-744 activated the adenosine monophosphate-activated protein kinase (AMPK) signaling pathway, and to a lesser extent, mammalian target of rapamycin (mTOR) signaling. The study provides evidence of a critical role for miR-744 in $\mathrm{PCa}$,and targeting miR-744 may represent a potential target for $\mathrm{PCa}$.

\section{Materials and methods}

Clinical tissues. All PCa tissues and adjacent tissues were collected from surgical resections from patients with $\mathrm{PCa}$ at the China and Japan Union Hospital of Jilin University (Changchun. China). Informed consent was received from all patients, and the research methodology was approved by the Ethics Committee of Jilin University. All tissue samples were immediately frozen and preserved in liquid nitrogen until use.

Cell culture. The PCa cell lines PC3 (ATCC ${ }^{\circledR}$ CRL-7934), Du145 (ATCC ${ }^{\circledR}$ HTB-81), and PPC-1 (ATCC ${ }^{\circledR}$ HTB-190) were obtained from The American Type Culture Collection (Manassas, VA, USA). The non-tumorigenic human prostate epithelial cell line 9 (NHP9) was procured from Dr. Tang's lab. Cells were cultured in Dulbecco's modified Eagle's medium (HyClone; GE Healthcare Life Sciences, Logan, UT, USA) containing $10 \%$ (v) heat-inactivated fetal bovine serum (Invitrogen; Thermo Fisher Scientific, Inc., Waltham, MA, USA) at $37^{\circ} \mathrm{C}$ in a humidified incubator with $5 \%(\mathrm{v} / \mathrm{v}) \mathrm{CO}_{2}$.

Cell transfection. The sequences of the miR-744 mimic, its inhibitor, and the corresponding negative control, were obtained from GenePharma Co., Ltd. (Shanghai, China). A total of $2 \times 10^{3}$ PC 3 cells were seeded into $35-\mathrm{mm}$ plates and incubated for $24 \mathrm{~h}$, and Lipofectamine ${ }^{\circledR} 2000$ (Invitrogen; Thermo Fisher Scientific, Inc.) was used to transfect miRNA-744 the cells. miRNAs were used at a concentration of $50 \mathrm{nM}$. Following a $6 \mathrm{~h}$ incubation, cells were transferred to complete medium, and cell lysates were harvested $48 \mathrm{~h}$ post transfection. Their sequences were as follows: Mimics-NC, 5'-UUCUCCGAA CGUGUCACGUTT-3' and 3'-ACGUGACACGUUCGGAGA ATT-5'; miR-744, 5'-UGCGGGGCUAGGGCUAACAGCA-3' and 3'-CUGUUAGCCCUAGCCCCGCAUU-5'; inhibitor-NC, 5'-CAGUACUUUUGUGUAGUACAA-3' and miR-744 inhibitor, 5'-UGCUGUUAGCCCUAGCCCCGCA-3'.

$R N A$ extraction and reverse transcription-quantitative polymerase chain reaction $(R T-q P C R)$. Total RNA was extracted from $\mathrm{PCa}$ tumor samples and their corresponding adjacent tissue samples or from PCa cell lines using TRIzol ${ }^{\circledR}$ (Invitrogen; Thermo Fisher Scientific, Inc.). All methods were performed according to the manufacturer's protocol. RNA was reverse transcribed into cDNA using Superscriptase II (Invitrogen; Thermo Fisher Scientific, Inc.). qPCR was performed using the Power SYBR Green PCR master mix (Life Technologies; Thermo Fisher Scientific, Inc.) according to the manufacturer's protocol. The thermocycling conditions were as follows: 35 cycles of $95^{\circ} \mathrm{C}$ for $30 \mathrm{sec}, 60^{\circ} \mathrm{C}$ for $30 \mathrm{sec}$, and $72^{\circ} \mathrm{C}$ for $35 \mathrm{sec}$. The following primer sets were used to measure the expression of LKB1: LKB1 forward, 5'-TGTCGG
TGGGTATGGACAC-3' and reverse, 5'-CCTTGCCGTAAG AGCCTTCC-3'; GAPDH-forward, 5'-AGCCTCCCGCTT CGCTCTCT-3' and reverse, 5'-GCGCCCAATACGACCAAA TCCGT-3'. GAPDH was used for normalization.

To detect miR-744 expression by qPCR, a hairpin-it ${ }^{\mathrm{TM}}$ miRNA qPCR quantitation kit (GenePharma Co., Ltd, Shanghai, China) was used. In brief, total RNA was reverse transcribed using miR-744 specific stem-loop RT primers, and levels of miR-744 measured. A miR-744 specific molecular beacon probe was used to ensure the accuracy of the real-time PCR data. The $2^{-\Delta \Delta C q}$ method was used to calculate relative expression levels (9). The following primer sets were used to measure the expression of LKB1 miR-744 Forward primer, 5'-ACACTCCAGCTGGGTGCGGGGCTAGGGCTA AC-3'; GAPDH-forward, 5'-AGCCTCCCGCTTCGCTCT CT-3' and reverse, 5'-GCGCCCAATACGACCAAATCCGT-3'.

Antibodies and western blotting. PC3 cells were lysed in ice-cold radioimmunoprecipitation assay buffer (Beyotime Institute of Biotechnology, Haimen, China) containing a protease inhibitor cocktail (Roche Diagnostics, Basel, Switzerland). The protein concentration of the lysates was measured using a bicinchoninic acid assay kit (Pierce; Thermo Fisher Scientific, Inc.). Equal amounts of each protein $(40 \mu \mathrm{g})$ were separated by $12 \%$ SDS-PAGE and transferred to a nitrocellulose membrane (Pall Life Sciences, Port Washington, NY, USA). The membranes were blocked with $5 \%$ nonfat milk for $2 \mathrm{~h}$ at room temperature, and incubated with primary antibodies against $\beta$-actin $(1: 2,000$; cat. no. 3700$)$, cyclin D1 (1:1,000; cat. no. 2922), cyclin-dependent kinase 4 (CDK4) (1:500; cat. no. 12790), proliferating cell nuclear antigen (PCNA) (1:1,000; cat. no. 2586), AMPK $\alpha$ (1:500; cat. no. 5831), AMPK $\beta 1$ (1:1,000; cat. no. 12063), phospho-AMPK $\alpha$ (Thr172) (1:200; cat. no. 2535), phospho-AMPK $\beta 1$ (Ser182) (1:250; cat. no. 4186) TSC2 (1:500; cat. no. 3990), phospho-TSC2 (Ser1387) (1:250; cat. no. 23402), mTOR (1:1,000; cat. no. 2983), phospho-mTOR (Ser2448) (1:250 dilution; cat. no. 2971) acquired from Cell Signaling Technology, Inc., (Danvers, MA, USA), and p21 $1^{\text {Cip1/Wafl }}$ (1:500; cat. no. 610233; BD Pharmingen; Becton, Dickinson and Company, Franklin Lakes, NJ, USA) at $4^{\circ} \mathrm{C}$, over night. After washing and incubating with rabbit (cat. no. 926-32201) or mouse (cat. no. 926-32211) secondary antibodies $(1: 10,000)$ dilution (LI-COR Biosciences, Lincoln, $\mathrm{NE}, \mathrm{USA}$ ) for $1 \mathrm{~h}$ at room temperature, the blots were imaged using the LI-COR imaging system, and band density was quantified using Odyssey 3.0 software (both LI-COR Biosciences, Lincoln, NE, USA) for each group, and normalized to $\beta$-actin.

Cell Counting kit-8 (CCK-8) cell viability assay. PC3 cells were transfected with an miR-744 inhibitor or negative control sequences respectively (outlined above). A total of $24 \mathrm{~h}$ post-transfection, cells were transferred into 96-well plates at a density of $2 \times 10^{3}$ cells/well for a further 48 h. Cell viability was assessed using the CCK- 8 assay per the manufacturer's protocol (Dojindo Molecular Technologies, Inc., Kumamoto, Japan).

Acridine orangelethidium bromide (AO/EB) fluorescence staining. PC3 cells $\left(2 \times 10^{3}\right)$ in the exponential growth phase were cultured on sterile coverslips for $24 \mathrm{~h}$, followed by transfection with miR-744 inhibitor or negative control sequences, 

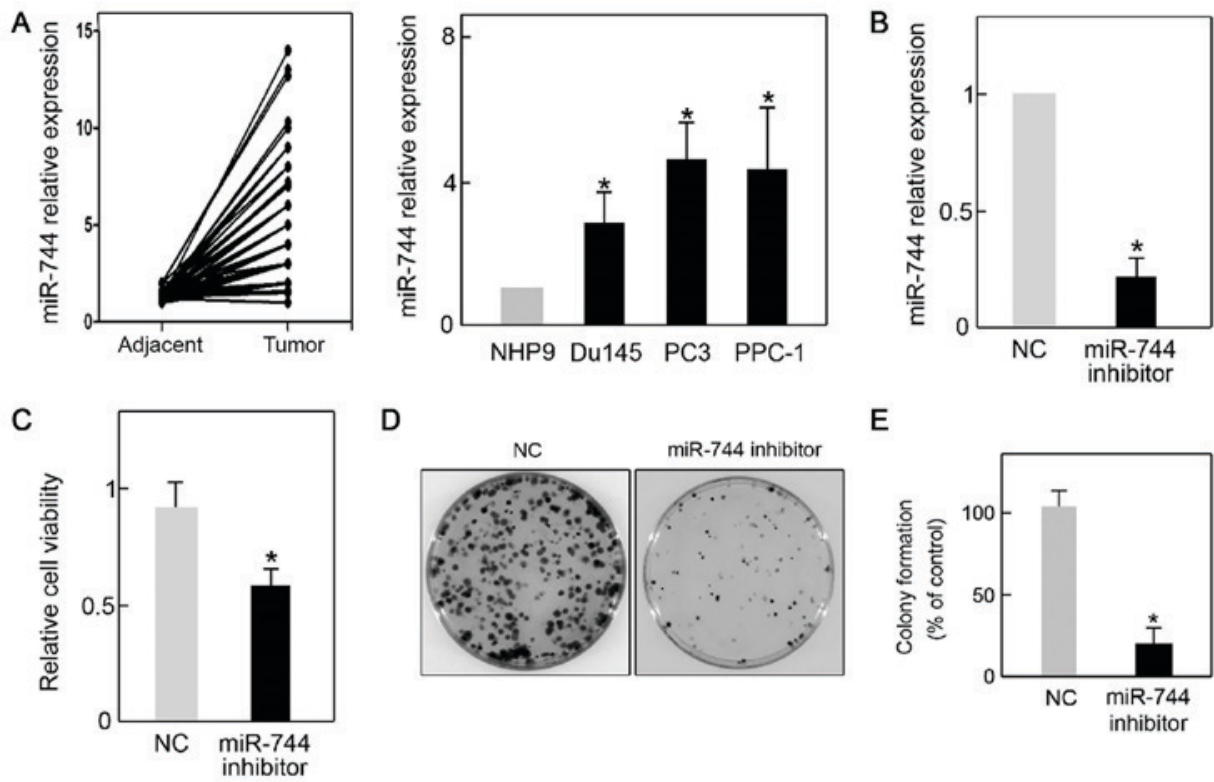

D

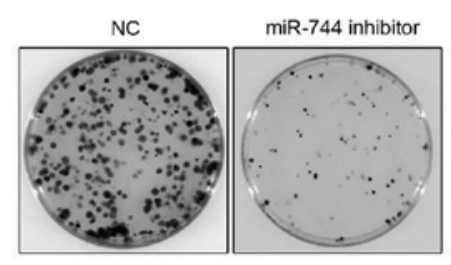

E
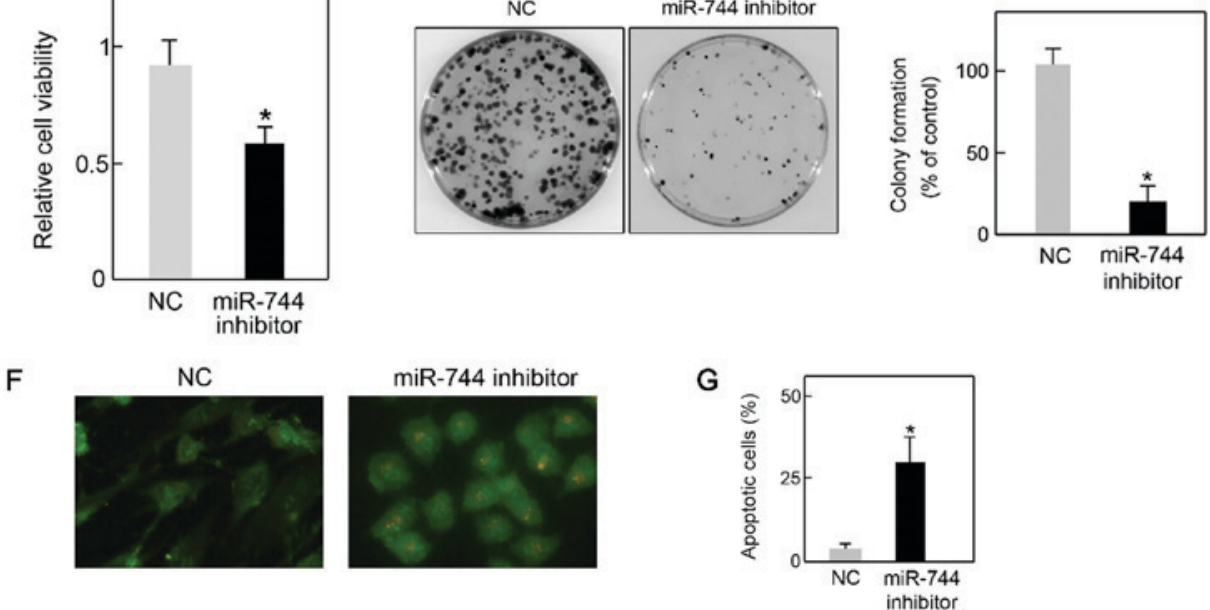

Figure 1. miR-744 is upregulated in PCa, and promotes PCa cell growth. (A) miR-744 is overexpressed in PCa tissues and cell lines. Reverse transcription-quantitative polymerase chain reaction was performed to measure the expression level of miR-744. (B) miR-744 inhibitor successfully reduced miR-744 levels in PC3 cells. (C) PC3 cells transfected with miR-744 inhibitor or NC sequence were cultured for $48 \mathrm{~h}$, and cell viability was 1analyzed using the CCK-8 assay. (D) miR-744 knockdown decreased PC3 colony formation capability to (E) a statistically significant degree. (F) miR-744 knockdown increased the apoptotic cell population in PC3 cells (x200 magnification) to (G) a statistically significant degree. ${ }^{*} \mathrm{P} \leq 0.05$ vs. respective $\mathrm{NC}(\mathrm{n}=3$ ). Prostate cancer, $\mathrm{PCa}$; miR, microRNA; NC, negative control; PCa, prostate cancer.

and were incubated with AO/EB mix solution for 5 min per the manufacturer's protocol (Beijing Solarbio Science \& Technology Co., Ltd, Beijing, China). Cell morphology was examined using a fluorescence microscope at magnification $\times 200$, and the percentage of apoptotic cells was calculated using the following formula: Apoptotic rate $(\%)=($ number of apoptotic cells/number of total cells) x100.

Immunofluorescence assay. PC 3 cells $\left(8 \times 10^{2}\right)$ were seeded onto cover slips and transfected with miR-744 inhibitor or negative control sequences. After $48 \mathrm{~h}$, cells were fixed and incubated with Ki-67 antibody (cat. no. 11882, 1:200, Cell Signaling Technology, Inc.) for $1 \mathrm{~h}$ and subsequently incubated with Alexa-488-Fluor (Thermo Fisher Scientific, Inc.) at room temperature for $20 \mathrm{~min}$ in the dark. Cells were then counterstained with DAPI $(1: 1,000)$ for $5 \mathrm{~min}$ to stain the cell nucleus. All coverslips were mounted using Prolong ${ }^{\circledR}$ diamond antifade mounting reagent (Applied Biosystems; Thermo Fisher Scientific, Inc.).

Dual luciferase reporter assay. Wild-type (WT) LKB1 (LKB1-WT) and mutant LKB1 (LKB1-Mut) 3'-UTR were cloned into separate pMIR-REPORT Luciferase vectors (Ambion; Thermo Fisher Scientific, Inc.). PC3 cells $\left(2 \times 10^{3}\right)$ were harvested from six-well plates and treated with the specific vectors and Lipofectamine 2000 (Invitrogen; Thermo Fisher Scientific, Inc.) for $48 \mathrm{~h}$. Luciferase activity was assessed by the Dual Luciferase-reporter 1000 assay system (Promega Corporation, Madison, WI, USA). Renilla activity was used for normalization.

Kaplan-Meier method. All clinical data and the Tier 3 RNASeqV2 mRNA expression data were downloaded from https://cancergenome.nih.gov. Samples for patients with a follow up time or time to mortality $>0$ days were kept for analysis. For each gene, all samples were divided into two groups (low and high expressing groups) based on median expression values. Kaplan-Meier analysis was performed to test the significance between the two groups.

Cox proportional hazards regression was also performed with the coxph function from the $\mathrm{R}$ survival library (https://cran.r-project.org/web/packages/survival/index.html). Hazard ratios with $95 \%$ confidence intervals were obtained.

Statistical analysis. Data are presented as the mean \pm standard error of the mean from three independent experiments. Statistical analysis was conducted using SPSS 19.0 software (IBM Corp., Armonk, NY, USA) and illustrated using GraphPad Prism 5.0 (GraphPad Software, La Jolla, CA, USA). Statistical significance was determined using the Student's t-test to compare two groups or analysis of variance with Tukey's post-hoc test to compare multiple groups. Paired Student's t-test was used to analyze the results. Pearson's correlation was used to assess the correlation between miR-744 and 
LKB1 expression. $\mathrm{P} \leq 0.05$ was considered to indicate a statistically significant difference.

\section{Results}

miR-744 is upregulated in PCa and promotes PCa cell growth. To verify the expression of miR-744 in PCa, tumor tissue samples and their corresponding adjacent tissue samples were isolated from 60 individual PCa patients, and levels of miR-744 were quantified by qPCR. As presented in Fig. 1A, cancer tissues demonstrated a significantly higher level of miR-744 expression when compared with adjacent tissues, suggesting a potential role for miR-744 in PCa. In agreement with this was the finding that following quantification of miR-744 levels in three PCa cell lines (PC3, Du145 and PPC-1), miR-744 levels were higher in $\mathrm{PCa}$ cells when compared with the non-tumorigenic prostate epithelial cell line NHP9 (Fig. 1A). However, PC3 cells expressed the highest level of miR-744, when compared with the other PCa cell lines. For this reason, PC3 cells were used for the remainder of the in vitro study. To elucidate the biological function of miR-744 in PCa, PC3 cells were transfected with miR-744 inhibitor or its negative control sequence (NC) respectively. Using qPCR, it was confirmed that the miR-744 inhibitor successfully reduced the level of miR-744 in PC3 cells (Fig. 1B). Furthermore, the capacity for cell growth was assessed with the CCK-8 and colony formation assay. The results demonstrated that cell viability was significantly reduced by miR-744 knockdown (Fig. 1C). miR-744 inhibitor transfection also resulted in a lower number of colonies (Fig. 1D and E). Unexpectedly, it was observed that silencing of miR-744 led to an increased population of apoptotic cells as measured by AO/EB staining (Fig. 1F and G). These data demonstrated that miR-744 was overexpressed in PCa cells and promoted PCa cell growth.

miR-744 downregulation inhibits cell proliferation. To confirm the role of miR-744 in the regulation of cell proliferation, PC3 cells were transfected with an miR-744 inhibitor and its NC respectively, and the cells assessed using Ki-67 immunofluorescence. A lower Ki-67 immunofluorescence was demonstrated in PC3 cells following miR-744 knockdown, indicating a lower proliferative capacity (Fig. 2A). Cell cycle analysis using flow cytometry revealed that silencing of miR-744 resulted in a larger population of cells in the G1 phase of the cell cycle and a smaller population in the $S$ phase (Fig. 2B). Cell cycle associated proteins, cyclin D1, CDK4, PCNA, and cyclin-dependent kinase inhibitor p21 (p21) were measured by western blotting, and the results demonstrated that levels of cyclin D1, CDK4, and PCNA were all reduced, whereas the cell cycle inhibitor p21 was increased (Fig. 2C and D). These data indicated that miR-744 knockdown inhibits PC3 cell proliferation.

miR-744 directly targets LKB1. To determine the association between miR-744 and LKB1, the expression of LKB1 in PCa tissue samples and their corresponding adjacent samples, and in PCa cell lines was confirmed (Table I). Using qPCR, it was revealed that the expression of LKB1 was lower in $\mathrm{PCa}$ tissues than in the adjacent tissues (Fig. 3A). Additionally, lower expression of LKB1 in PCa cell lines PC3, Du145 and
Table I. Patient clinical information.

\begin{tabular}{lc}
\hline Variable & No. patients $(\mathrm{n}=60)$ \\
\hline Age, years [median (range)] & $45.3(30-85)$ \\
Sex, female & $30(50 \%)$ \\
Tumor size, $\leq 5 \mathrm{~cm}$ & $40(66.7 \%)$ \\
Tumor size, $>5 \mathrm{~cm}$ & $20(33.3 \%)$ \\
Lymph node involvement & $15(25 \%)$ \\
Metastasis & 55 (negative) $/ 5$ (positive)
\end{tabular}

PPC-1 was observed when compared with non-tumorigenic prostate epithelial (NHP9) cells (Fig. 3B). The expression of miR-744 was also assessed following transfection with an miR-744 mimic. The expression of miR-744 was found to be upregulated in the miR-744 mimic group compared with the $\mathrm{NC}$ group (Fig. 3C). It is known that miRNA regulates gene expression by targeting the 3'-UTR of target mRNA, leading to its degradation or translational inhibition. By consulting bioinformatics databases (www.targetScan.org), LKB1 was revealed to be a potential target of miR-744 (Fig. 3D). The qPCR results demonstrated that LKB1 mRNA expression level was negatively associated with the level of miR-744 (Fig. 3E and F). Additionally, the protein levels of LKB1 showed a similar negative association pattern with miR-744 to that of the mRNA expression levels (Fig. 3G and H). To address whether LKB1 was a direct target of miR-744, a dual luciferase assay was performed. Co-transfection with miR-744 mimics/LKB1-WT resulted in a reduction in luciferase activity, whereas miR-744 mimics did not alter the luciferase activity in the LKB1 mutant group (Fig. 3I). All results indicated that LKB1 is a direct target of miR-744.

miR-744 downregulation interferes with AMPK signaling and inhibits cell growth. LKB1 is well established as a potent tumor suppressor involved in the activation of the AMPK signaling pathway. Following evidence that LKB1 is a direct target of miR-744, it was hypothesized that miR-744 may regulate AMPK signaling. Western blot analysis demonstrated that the phosphorylation of T172 on AMPK $\alpha$ and S182 on AMPK $\beta$ was higher following miR-744 knockdown, suggesting that the AMPK signaling pathway was activated (Fig. 4A and B). The tumor suppressor Tuberin (TSC2) is a downstream target of AMPK signaling, and the activation of AMPK signaling by miR-744 knockdown resulted in TSC2 and S1387 phosphorylation (Fig. 4C). The mTOR signaling pathway may be negatively regulated by AMPK signaling. Therefore, mTOR signaling activity was determined following miR-744 knockdown. Notably, knockdown reduced mTOR signaling in PC3 cells, as determined by its phosphorylation of S2448 (Fig. 4D). To strengthen the data, PC3 cells were transfected with miR-744 mimics and the activation of AMPK and mTOR signaling pathways determined; miR-744 mimics successfully blocked the AMPK signaling pathway, and activated mTOR signaling (Fig. 4E-H).

Knockdown of LKBIp reverses the effect of miR-744 silencing in PC3 cells. To examine the association between miR-744 

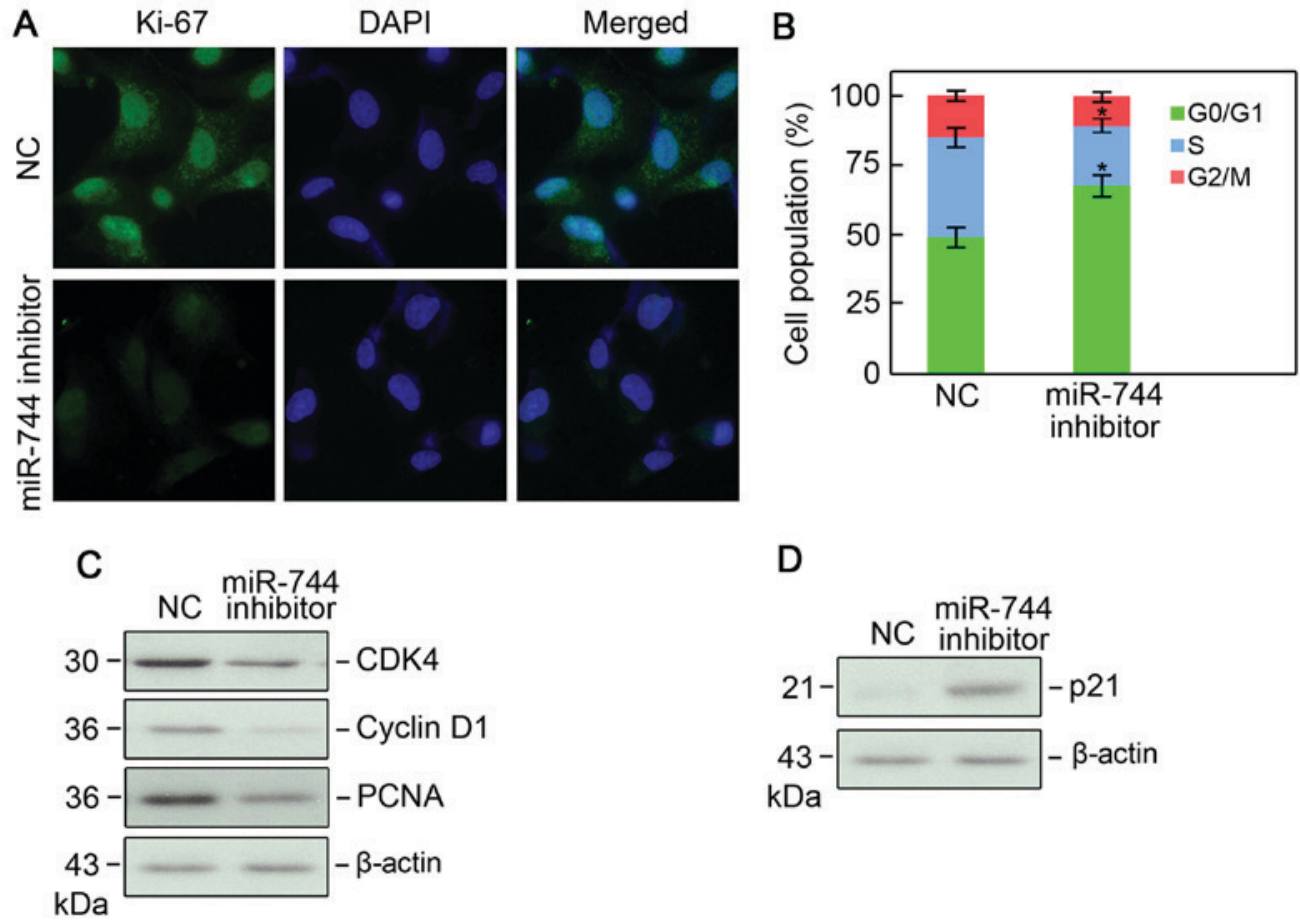

D

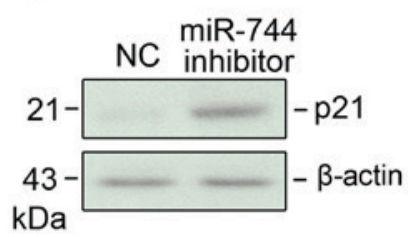

Figure 2. miR-744 downregulation inhibits cell proliferation. (A) miR-744 knockdown significantly decreased PC3 cell proliferation as determined by the Ki-67 immunofluorescence assay (magnification x200). (B) Cell cycle profiles analyzed by flow cytometry. (C) Alterations in cyclin-dependent kinase 4 , cyclin D1, and proliferating cell nuclear antigen protein levels detected by western blotting. (D) The protein level of p21 was increased upon miR-744 knockdown. ${ }^{*} \mathrm{P} \leq 0.05$ vs. respective $\mathrm{NC}$ ( $\mathrm{n}=3$ independent experiments for each condition). miR, microRNA; CDK4, cyclin-dependent kinase 4; PCNA, proliferating cell nuclear antigen; p21, cyclin-dependent kinase inhibitor p21; NC, negative control.

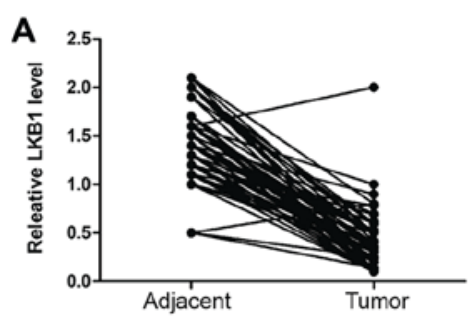

D
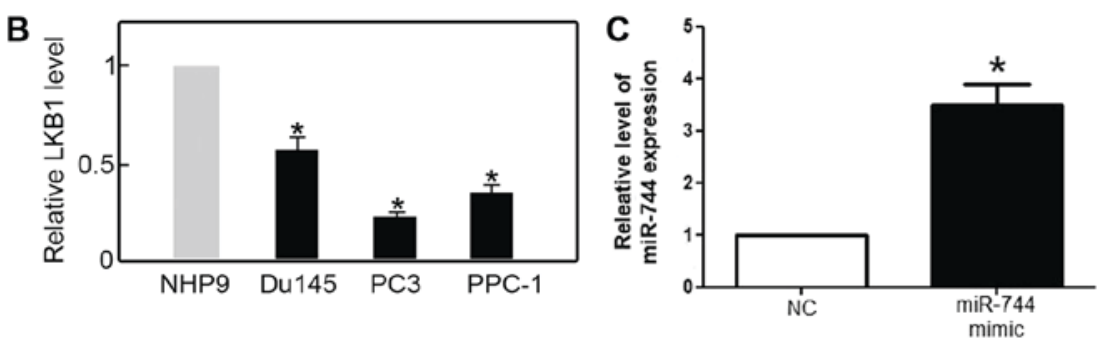

E
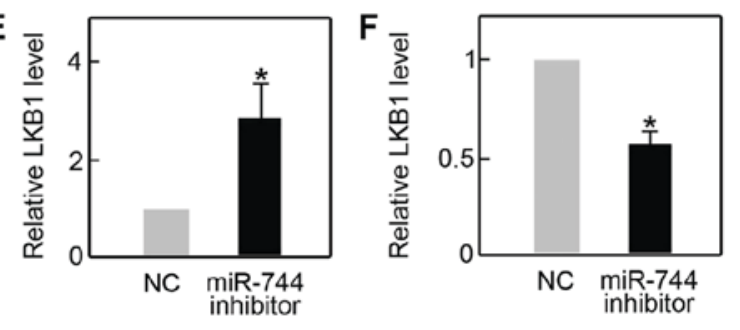

H

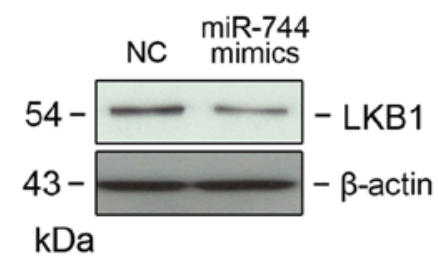

I

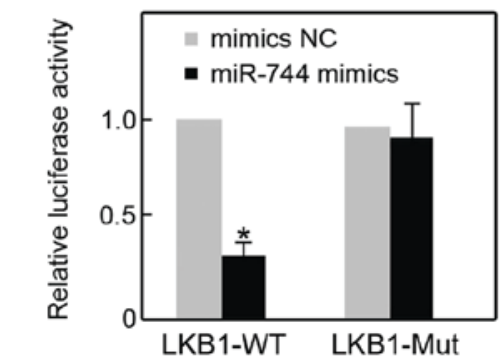

Figure 3. miR-744 directly targets LKB1. (A) LKB1 was downregulated in PCa tissues and (B) cell lines. (C) The expression of miR-744 following transfection with miR-744 mimic. (D) Schematic diagram of the miR-744 binding site on LKB1. LKB1 mRNA levels were negatively associated with miR-744 levels, as measured by RT-qPCR; observed at low (E) and high (F) LKB1 mRNA levels. LKB1 protein levels were negatively correlated to miR-744 levels, as detected by western blotting; observed at low (G) and high (H) LKB1 protein levels. (I) Luciferase reporter assay. PC3 cells were co-transfected with a luciferase construct fused to the LKB1-WT or LKB1-MUT 3'-UTR and miR-744 mimic or mimics NC. The relative luciferase activity was detected. "P $\leq 0.05$ vs. respective NC (n=3). LKB1, liver kinase B1; miR, microRNA; NC, negative control; LKB1-WT, liver kinase B1- wild type; LKB1-MUT, liver kinase B1-mutant. 
A
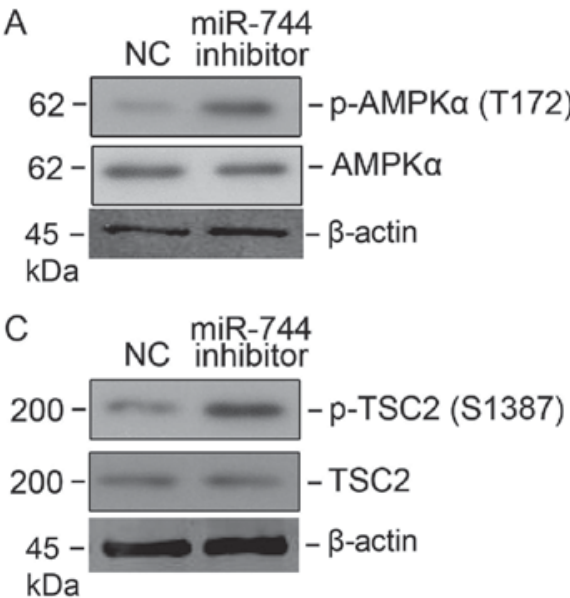

$\mathrm{E}$
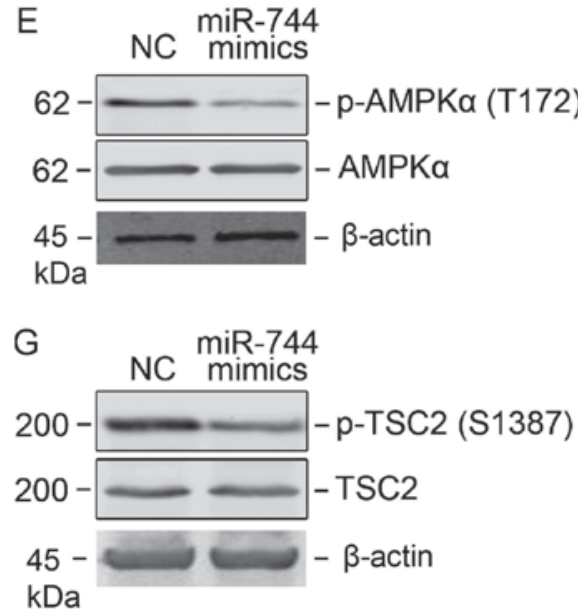

B $\quad$ miR-744

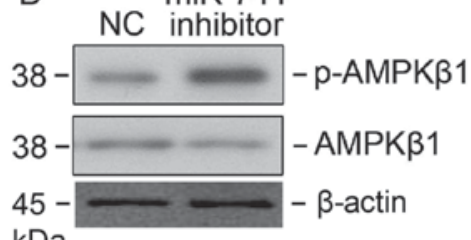

$\mathrm{kDa}$

D NC miR-744

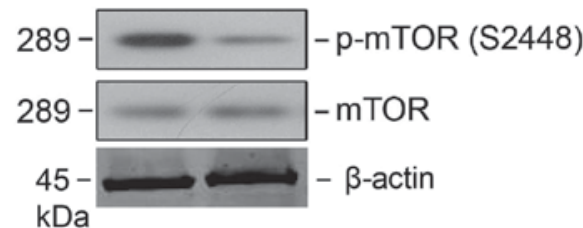

F NC $\begin{gathered}\text { miR-744 } \\ \text { mimics }\end{gathered}$

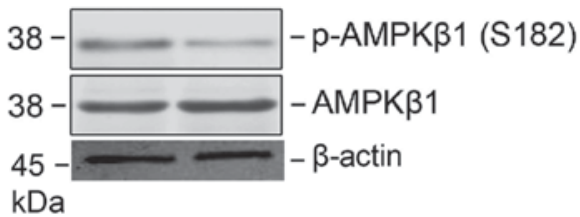

H NC miR-744

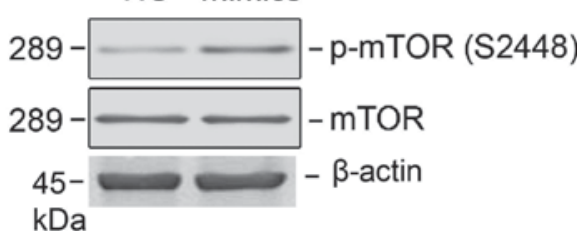

Figure 4. miR-744 interferes with AMPK signaling to inhibit cell growth. miR-744 knockdown activated the AMPK signaling pathway as determined by (A) AMPK $\alpha$, and (B) AMPK $\beta 1$ phosphorylation. (C) miR-744 knockdown regulated TSC2 activity by phosphorylation at S1387. (D) miR-744 knockdown inhibited mTOR activation. Overexpression of miR-744 inhibited the AMPK signaling pathway as determined by (E) AMPK and (F) AMPK $\beta 1$ phosphorylation. (G) Upregulation of miR-744 regulated TSC2 inhibition by phosphorylation at S1387. (H) Overexpression of miR-744 caused mTOR activation. AMPK, adenosine monophosphate-activated protein kinase; miR, microRNA; TSC2, tuberin; mTOR, mammalian target of rapamycin; NC, negative control.

A

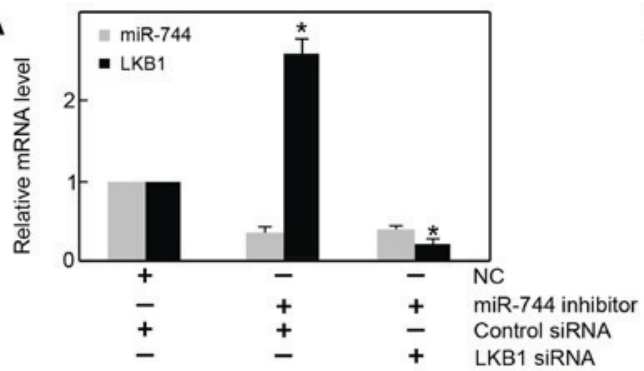

B

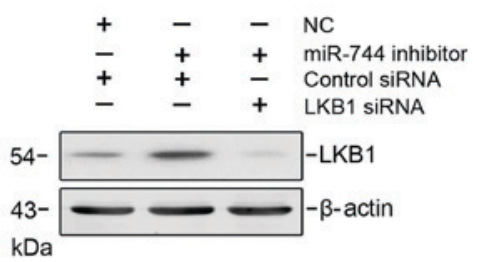

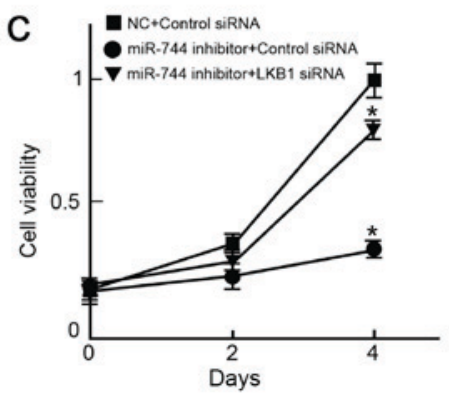

D

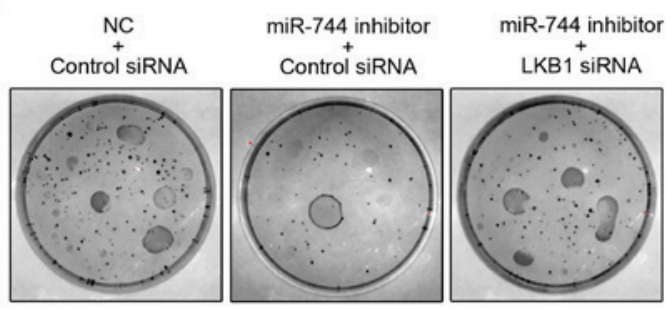

Figure 5. Knockdown of LKB1 reverses the effects of miR-744 silencing in PC3 cells. Co-transfection of LKB1-siRNA successfully restored LKB1 expression in PC3 cells and downregulated miR-744 at the (A) miR and (B) protein level. (C) LKB1-siRNA reversed PC3 cell viability inhibition produced by miR-744 silencing. (D) LKB1-siRNA reversed the reduction in colony formation capability induced by miR-744 downregulation. "P $\leq 0.05$ vs. respective NC (n=3). LKB1, liver kinase B1; miR, microRNA; siRNA, small interfering RNA; NC, negative control. 

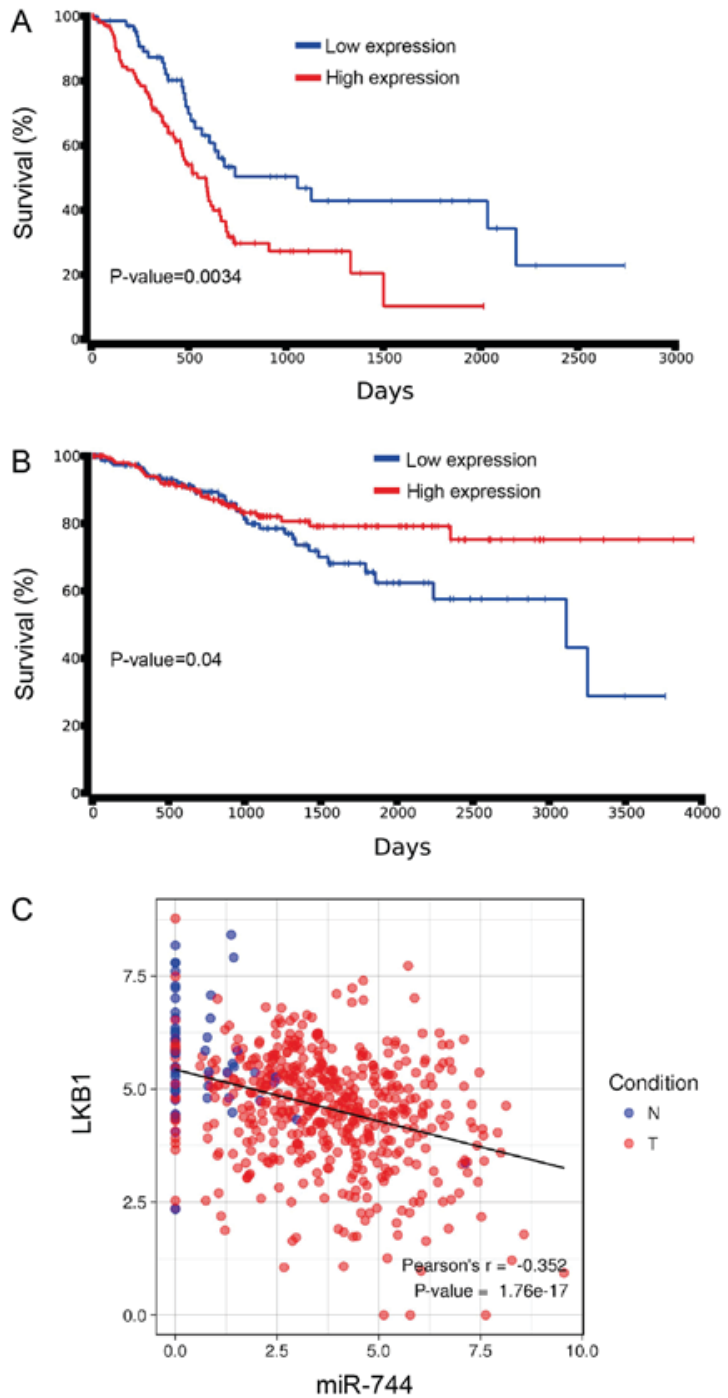

Figure 6. miR-744 and LKB1 are involved in PCa patient survival. The Kaplan-Meire survival analysis of (A) miR-744 and (B) LKB1. (C) Expression correlation between LKB1 and miR-744 in the TCGA PCa dataset. LKB1, liver kinase $\mathrm{B} 1$; miR, microRNA; N, normal samples; $\mathrm{T}$, PCa samples.

and LKB1 in PCa cells, the LKB1 expression vector was co-transfected with miR-744. It was verified that LKB1 expression was restored by co-transfection of the LKB1 expression vector (Fig. 5A and B), and it was revealed that the viability of PC3 cells was preserved when analyzed with the CCK-8 and clonogenic assays (Fig. 5C and D).

miR-744 and LKB1 are involved in the survival of $P C a$ patients. To investigate the potential role of miR-744 and LKB1 in PCa survival, the Kaplan-Meier test was used to determine the correlation between overall survival times in patients with miR-744, and LKB1 expression in PCa patients, using data from the TCGA database. Patient survival was lower with increased miR-744 expression, whereas survival was increased in those patients exhibiting negative or low- miR-744 expression (Fig. 6A). However, with lower LKB1 expression, patient survival was significantly longer compared with patients with higher LKB1 expression (Fig. 6B). A similar negative correlation was observed between LKB1 and miR-744 in the TCGA PCa patient database (Fig. 6C).

\section{Discussion}

$\mathrm{PCa}$ is clinically challenging to treat. Therefore, understanding the molecular mechanisms of $\mathrm{PCa}$ carcinogenesis and progression has become crucial for the development of effective new therapies. Non-coding RNAs, particularly miRNAs, have opened up novel research avenues for PCa $(19,20)$. It has been reported that expression levels of numerous miRNAs are altered in PCa (21). This alteration indicates a potential role for miRNAs in PCa progression. Researchers have determined that miRNAs are involved in all processes associated with cancer, including oncogenesis, migration, invasion and angiogenesis (22-24). The present study focused on miR-744 and investigated its biological function and mechanism of action in PCa. It was identified that the expression of miR-744 was upregulated in $\mathrm{PCa}$ tissues. Knockdown of miR-744 produced PC3 cell growth inhibition, and increased apoptosis. Furthermore, knockdown of miR-744 reduced the capability of PC3 cells to proliferate. The involvement of miR-744 and LKB1 in PCa patient survival was also demonstrated. It has been reported that miR-744 promotes $\mathrm{PCa}$ progression through activation of Wnt/ $\beta$-catenin signaling (25). The findings from the present study are consistent with those of the previous studies, and have revealed a potential mechanism of miR-744 in $\mathrm{PCa}$ progression, involving the suppression of LKB1.

To date, miRNAs have been known to suppress target gene expression by binding to the 3'-UTR of target mRNAs and initiating the degradation of target sequences or inhibiting translation (26). Multiple genes, such as tyrosine-protein phosphatase non-receptor type 1, ARHGAP5, programmed cell death protein 4 and elongation factor 1-alpha 2 have been identified and validated as targets for miR-744 (17,27-29). The present study demonstrates that the tumor suppressor LKB1 is a potential target of miR-744. RT-qPCR and western blot analysis confirmed that LKB1 expression negatively correlates with miR-744 levels. This expression pattern was consistent with the classical miRNA regulation model. The dual luciferase assay further confirmed that LKB1 is directly targeted by miR-744.

LKB1 is a well-established tumor suppressor that activates the AMPK signaling pathway $(30,31)$ by directly phosphorylating T172 on AMPK, a requirement for its activation (32). As LKB1 was identified as a direct target of miR-744, activation of AMPK signaling was further investigated following miR-744 knockdown. Elevated phosphorylation of AMPK was observed, suggesting activation of the AMPK signaling pathway. Crosstalk between the mTOR and the AMPK signaling pathways is widely accepted (33). The AMPK signaling pathway may inhibit mTOR signaling through TSC2, and thereby promote the arrest of cancer cell proliferation $(34,35)$. Higher TSC2 phosphorylation and lower mTOR phosphorylation were consistently detected in miR-744 silenced PC3 cells. These results indicate that the mTOR signaling pathway is inhibited by miR-744 knockdown. Therefore, it was concluded that miR-744 may regulate PCa cancer cell growth, at least through the AMPK signaling and mTOR signaling pathways.

Taken together, these findings demonstrate a novel insight into the biological function of miR-744 in PCa, and 
its molecular mechanism of action. Our findings suggest that miR-744 may serve an oncogenic role in $\mathrm{PCa}$, and that it may be a potential therapeutic target for PCa treatment.

\section{Acknowledgements}

The authors would like to thank Dr G. Tang (Department of Molecular Carcinogenesis, The University of Texas MD Anderson Cancer Center) for his donation of human prostate epithelial cell line 9 (NHP9).

\section{Funding}

No funding was received.

\section{Availability of data and materials}

The datasets used during the present study are available from the corresponding author upon reasonable request.

\section{Authors' contributions}

HYL and MZ designed the study and analyzed and interpreted the cell experiment data and patient data regarding the prostate cancer. HL and YZ performed the RT-qPCR experiments. MZ and $\mathrm{YZ}$ were major contributors in writing the manuscript. All authors read and approved the final manuscript. All authors were involved in the conception of the study, read and approved the manuscript and $\mathrm{MZ}$ and $\mathrm{YZ}$ agree to be accountable for all aspects of the research in ensuring that the accuracy or integrity of any part of the study are appropriately investigated and resolved.

\section{Ethics approval and consent to participate}

Informed consent was received from all patients, and the research methodology was approved by the Ethics Committee of Jilin University (Changchun, China).

\section{Patient consent for publication}

All the patient, or parent, guardian or next of kin (in case of deceased patients) have provided written informed consent for the publication of any associated data and accompanying images.

\section{Competing interests}

The authors declare that they have no competing interests.

\section{References}

1. Siegel R, Ma J, Zou Z and Jemal A: Cancer statistics, 2014. CA Cancer J Clin 64: 9-29, 2014

2. Jemal A, Bray F, Center MM, Ferlay J, Ward E and Forman D: Global cancer statistics. CA Cancer J Clin 61: 69-90, 2011.

3. Chen W, Zheng R, Zeng H, Zhang S and He J: Annual report on status of cancer in China, 2011. Chin J Cancer Res 27: 2-12, 2015.

4. Pound CR, Partin AW, Eisenberger MA, Chan DW, Pearson JD and Walsh PC: Natural history of progression after PSA elevation following radical prostatectomy. JAMA 281: 1591-1597, 1999.
5. Xie H, Li C, Dang Q, Chang LS and Li L: Infiltrating mast cells increase prostate cancer chemotherapy and radiotherapy resistances via modulation of p38/p53/p21 and ATM signals. Oncotarget 7: 1341-1353, 2016.

6. Sutherland SI, Pe Benito R, Henshall SM, Horvath LG and Kench JG: Expression of phosphorylated-mTOR during the development of prostate cancer. Prostate 74: 1231-1239, 2014.

7. Galardi S, Mercatelli N, Farace MG and Ciafrè SA: NF-kB and c-Jun induce the expression of the oncogenic miR-221 and miR-222 in prostate carcinoma and glioblastoma cells. Nucleic Acids Res 39: 3892-3902, 2011.

8. David R: Small RNAs: miRNA machinery disposal. Nat Rev Mol Cell Biol 14: 4-5, 2013.

9. Xiao R, Li C and Chai B: miRNA-144 suppresses proliferation and migration of colorectal cancer cells through GSPT1. Biomed Pharmacother 74: 138-144, 2015.

10. Xue J, Chi Y, Chen Y, Huang S, Ye X, Niu J, Wang W, Pfeffer LM, Shao ZM, Wu ZH and Wu J: MiRNA-621 sensitizes breast cancer to chemotherapy by suppressing FBXO11 and enhancing p53 activity. Oncogene 35: 448-458, 2016.

11. Lewis H, Lance R, Troyer D, Beydoun H, Hadley M, Orians J, Benzine T, Madric K, Semmes OJ, Drake R, et al: miR-888 is an expressed prostatic secretions-derived microRNA that promotes prostate cell growth and migration. Cell Cycle 13: 227-239, 2014.

12. Jin M, Zhang T, Liu C, Badeaux MA, Liu B, Liu R, Jeter C, Chen X, Vlassov AV and Tang DG: miRNA-128 suppresses prostate cancer by inhibiting BMI-1 to inhibit tumor-initiating cells. Cancer Res 74: 4183-4195, 2014.

13. Xuan H, Xue W, Pan J, Sha J, Dong B and Huang Y: Downregulation of miR-221, -30d and -15a contributes to pathogenesis of prostate cancer by targeting Bmi-1. Biochemistry (Mosc) 80: 276-283, 2015.

14. Singh PK, Preus L, Hu Q, Yan L, Long MD, Morrison CD, Nesline M, Johnson CS, Koochekpour S, Kohli M, et al: Serum microRNA expression patterns that predict early treatment failure in prostate cancer patients. Oncotarget 5: 824-840, 2014.

15. Miyamae M, Komatsu S, Ichikawa D, Kawaguchi T, Hirajima S, Okajima W, Ohashi T, Imamura T, Konishi H, Shiozaki A, et al: Plasma microRNA profiles: Identification of miR-744 as a novel diagnostic and prognostic biomarker in pancreatic cancer. Br J Cancer 113: 1467-1476, 2015.

16. Song MY, Pan KF, Su HJ, Zhang L, Ma JL, Li JY, Yuasa Y, Kang D, Kim YS and You WC: Identification of serum microRNAs as novel non-invasive biomarkers for early detection of gastric cancer. PLoS One 7: e33608, 2012.

17. Fang Y, Zhu X, Wang J, Li N, Li D, Sakib N, Sha Z and Song W: MiR-744 functions as a proto-oncogene in nasopharyngeal carcinoma progression and metastasis via transcriptional control of ARHGAP5. Oncotarget 6: 13164-13175, 2015.

18. Zhou W, Li Y, Gou S, Xiong J, Wu H, Wang C, Yan H and Liu T: MiR-744 increases tumorigenicity of pancreatic cancer by activating Wnt/ $\beta$-catenin pathway. Oncotarget 6: 37557-37569, 2015.

19. Lin F, Lei S, Ma J, Shi L, Mao D, Zhang S, Huang J, Liu X, Ding D, Zhang Y and Zhang S: Inhibitory effect of jianpi-jiedu prescription-contained serum on colorectal cancer SW48 cell proliferation by mTOR-P53-P21 signalling pathway. Zhong Nan Da Xue Xue Bao Yi Xue Ban 41: 1128-1136, 2016 (In Chinese).

20. Jackson BL, Grabowska A and Ratan HL: MicroRNA in prostate cancer: Functional importance and potential as circulating biomarkers. BMC Cancer 14: 930, 2014.

21. Song $\mathrm{C}$, Chen $\mathrm{H}$, Wang $\mathrm{T}$, Zhang $\mathrm{W}$, Ru $\mathrm{G}$ and Lang J: Expression profile analysis of microRNAs in prostate cancer by next-generation sequencing. Prostate 75: 500-516, 2015.

22. Josson S, Gururajan M, Hu P, Shao C, Chu GY, Zhau HE, Liu C, Lao K, Lu CL, Lu YT, et al: miR-409-3p/-5p promotes tumorigenesis, epithelial-to-mesenchymal transition, and bone metastasis of human prostate cancer. Clin Cancer Res 20: 4636-4646, 2014.

23. Wei P, Qiao B, Li Q, Han X, Zhang H, Huo Q and Sun J: microRNA-340 suppresses tumorigenic potential of prostate cancer cells by targeting high-mobility group nucleosome-binding domain 5. DNA Cell Biol 35: 33-43, 2016.

24. Ji H, Li Y, Jiang F, Wang X, Zhang J, Shen J and Yang X: Inhibition of transforming growth factor beta/SMAD signal by MiR-155 is involved in arsenic trioxide-induced anti-angiogenesis in prostate cancer. Cancer Sci 105: 1541-1549, 2014.

25. Guan H, Liu C, Fang F, Huang Y, Tao T, Ling Z, You Z, Han X, Chen S, Xu B and Chen M: MicroRNA-744 promotes prostate cancer progression through aberrantly activating Wnt $/ \beta$-catenin signaling. Oncotarget 8: 14693-14707, 2017. 
26. Pencheva $\mathrm{N}$ and Tavazoie SF: Control of metastatic progression by microRNA regulatory networks. Nat Cell Biol 15: 546-554, 2013.

27. Zhang X, Han X, Tang Y, Wu Y, Qu B and Shen N: miR-744 enhances type I interferon signaling pathway by targeting PTP1B in primary human renal mesangial cells. Sci Rep 5: 12987, 2015.

28. Zeng H, Qu J, Jin N, Xu J, Lin C, Chen Y, Yang X, He X, Tang S, Lan $\mathrm{X}$, et al: Feedback activation of leukemia inhibitory factor receptor limits response to histone deacetylase inhibitors in breast cancer. Cancer Cell 30: 459-473, 2016.

29. Vislovukh A, Kratassiouk G, Porto E, Gralievska N, Beldiman C, Pinna G, El'skaya A, Harel-Bellan A, Negrutskii B and Groisman I: Proto-oncogenic isoform A2 of eukaryotic translation elongation factor eEF1 is a target of miR-663 and miR-744. Br J Cancer 108: 2304-2311, 2013.

30. Hezel AF and Bardeesy N: LKB1; linking cell structure and tumor suppression. Oncogene 27: 6908-6919, 2008.

31. Blagih J, Krawczyk CM and Jones RG: LKB1 and AMPK: Central regulators of lymphocyte metabolism and function. Immunol Rev 249: 59-71, 2012.

32. Shaw RJ, Kosmatka M, Bardeesy N, Hurley RL, Witters LA, DePinho RA and Cantley LC: The tumor suppressor LKB1 kinase directly activates AMP-activated kinase and regulates apoptosis in response to energy stress. Proc Natl Acad Sci USA 101: 3329-3335, 2004.
33. Agarwal S, Bell CM, Rothbart SB and Moran RG: AMP-activated protein kinase (AMPK) control of mTORC1 is p53- and TSC2-independent in pemetrexed-treated carcinoma cells. J Biol Chem 290: 27473-27486, 2015.

34. Jozwiak J, Jozwiak S, Grzela T and Lazarczyk M: Positive and negative regulation of TSC2 activity and its effects on downstream effectors of the mTOR pathway. Neuromolecular Med 7: 287-296, 2005

35. Chen MB, Zhang Y, Wei MX, Shen W, Wu XY, Yao C and Lu PH: Activation of AMP-activated protein kinase (AMPK) mediates plumbagin-induced apoptosis and growth inhibition in cultured human colon cancer cells. Cell Signal 25: 1993-2002, 2013.

This work is licensed under a Creative Commons Attribution-NonCommercial-NoDerivatives 4.0 International (CC BY-NC-ND 4.0) License. 